\title{
MOTION NEAR THE UNIT CIRCLE IN THE THREE-BODY PROBLEM
}

ROGER A. BROUCKE

Department of Aerospace Engineering, University of Texas, Austin, TX 78712

\section{Introduction}

Many of the important applications of the circular planar restricted problem of three bodies involve motion in the vicinity of the unit circle, (as defined in canonical units). It is then of interest to develop simplified models which are valid in this region. These models preserve the gross characteristics of the original system but they possess simpler equations of motion.

We will also show that several simplified models can be seen as a perturbation of a very well known simple linear system: the Clohessy-Wiltshire equations used by NASA in all their rendezvous operations. These are actually very close to the wellknown Hill problem. We will thus consider the Restricted problem as a perturbed Hill or Clohessy-Wiltshire problem. We also introduce the Clohessy-Wiltshire Lagrangian in polar coordinates.

Actually we use polar coordinates throughout, in a rotating frame with canonical units of length and time. In this frame, the larger primary is at the origin, while the smaller primary is at (1.0). We are especially interested in the motion near the unit circle, $(r=1)$. This type of situation has numerous applications, such as for instance the Trojan Asteroids and the classification of orbits near the triangular points (such as "tadpoles" and "horseshoes"; see Dermott and Murray, 1981a). Many extensive numerical investigations of the manifolds of periodic orbits in the vicinity of the triangular points were made in the sixties by Deprit (1966) and Deprit and Henrard (1969) as well as Deprit, Henrard, Palmore and Price (1969).

Another motivation that prompted us to perform a systematic study of simplified models of three-body problem is the work of Garfinkel $(1975,1977,1978)$ on the Ideal Resonance problem; (see also Giacaglia, 1976).

One of the newer applications is in the dynamics of the coorbital satellites of Saturn, discovered by the Voyager Spacecraft. The theory of the coorbitals was studied extensively, for instance by Dermott and Murray (1981b), by Harrington and Seidelmann (1981), by Yoder and Colombo as well as by Salo and Yoder (1988). The dynamics of the coorbitals is without doubt a unit-circle phenomenon, although the igeneral problemi is a better model than the Restricted problem of three bodies, (Konopliv, 1986).

One of the main results of the present paper is that we discovered a new symmetry property that exists in some of our simplified models; the symmetry with respect to the unit-circle. Using this symmetry theorem in the model described at the end of section 8 , with the potential (8.6), we were able to compute horseshoe periodic orbits as well as the long and short-period periodic orbits that exist around $L_{4}$ and $L_{5}$ as well as the short period orbits around $L_{3}$. It is actually an amazing 
result that many of our rather strongly mutilated models of the restricted problem still have three classical libration points $L_{3}, L_{4}$ and $L_{5}$. Some of these models should be investigated for the existence of Brownis conjectures.

\section{The Restricted Problem of Three Bodies}

The equations will be derived using polar coordinates in which $\mathrm{r}$ is measured from the larger primary and $\theta$ is an angle in the rotating $x y$ system.

The Lagrangian per unit mass is

$$
L=\frac{1}{2}\left(\dot{r}^{2}+r^{2} \dot{\theta}^{2}\right)+r^{2} \dot{\theta}^{2}+\frac{r^{2}}{2}+\frac{1}{r}+\mu\left[\frac{1}{d}-r \cos \theta-\frac{1}{r}\right] .
$$

where

$$
d^{2}=1+r^{2}-2 r \cos \theta \text {. }
$$

The above Lagrangian has been written in the form of two parts, the second part being proportional to the mass $\mu$ of the smaller primary (located at 1,0 ) which will often be considered as a small quantity:

If $\mu$ is set equal to zero, we obtain the Lagrangian of the classical Kepler problem, referred to a uniformly rotating frame of reference. So the Lagrangian made up of the first five terms will be called the Keplerian Lagrangian.

\section{Motion near theUnit Circle; the First Approximate Model}

In order to investigate motion in the vicinity of the unit circle, we will let $r=1+\varepsilon$ where $\varepsilon<<1$. In what follows we will thus expand the Lagrangian of the restricted problem, to different orders in $\varepsilon$. In some instances we will expand the 5 Keplerian terms to a different order from the 3 remaining perturbation terms in $\mu$. This will allow us to obtain a large variety of Lagrangians which may not be very accurate representations of the true restricted problem, but which may show us some important connections with other well-known dynamical systems, such as the simple pendulum or the Clohessy-Wiltshire system for instance.

In the expansions that will be made the three quantities $\varepsilon, \dot{\varepsilon}$ and $\dot{\theta}$ will usually be considered as small quantities of the same order of magnitude. The angle $\theta$ will be considered as a large unconstrained number. Let us first consider the expansion of the five Keplerian terms, say up to order 4 in the three variables $\varepsilon, \dot{\varepsilon}$ and $\dot{\theta}$. We have first of all

$$
=1-\varepsilon+\varepsilon^{2}-\varepsilon^{3}+\varepsilon^{4} .
$$

The expansion of the Keplerian Lagrangian is thus

$$
L=\left[\frac{1}{2}\left(\dot{\varepsilon}^{2}+\dot{\theta}^{2}\right)+2 \varepsilon \dot{\theta}+\frac{3}{2} \varepsilon^{2}\right]+\left[\varepsilon \dot{\theta}^{2}+\varepsilon^{2} \dot{\theta}-\varepsilon^{3}\right]\left[\frac{1}{2} \varepsilon^{2} \dot{\theta}^{2}+\varepsilon^{4}\right],
$$


where the different brackets contain respectively the terms of order 2 , order 3 and order 4. Note that we have dropped a term $\dot{\theta}$ which is an exact derivative; and that the linear terms in $\varepsilon$ canceled each other out. The Lagrangian consisting of the first four quadratic terms will be called the Clohessy-Wiltshire Lagrangian. It will be studied in more detail later.

The expression for the distance $d$ between the smallest primary (of mass $\mu$ ) and the massless satellite is given by equation (2.2).

Assuming that $r=1+\varepsilon$, it is easy to expand $1 / d$ in powers of $\varepsilon$. We start from the exact formula

$$
d^{2}=2(1-\cos \theta)\left(1+\varepsilon+\frac{\varepsilon^{2}}{2(1-\cos \theta)}\right) .
$$

The expression in the last pair of parentheses can now be expanded in $\varepsilon$, with the use of the binomial formula (to the second order in $\varepsilon$ ):

$$
\frac{1}{d}=\frac{1}{\sqrt{2(1-\cos \theta)}} \cdot\left[1-\frac{\varepsilon}{2}+\frac{\varepsilon^{2}}{8}\left(3-\frac{2}{1-\cos \theta}\right)\right] \text {. }
$$

\section{The Unperturbed Clohessy-Wiltshire Lagrangian}

A very interesting point in our present formulation of the restricted three-body problem in polar coordinates is its connection with the so-called Clohessy-Wiltshire equations which are well known in the space program, in relation with circular relative motions and rendezvous operations. If in the previous Lagrangians for the restricted problem, we neglect all the perturbation terms in $\mu$ and we keep only the terms up to order 2 in $\dot{\varepsilon}$ and $\dot{\theta}$, we obtain

$$
L=L_{C W}=\frac{1}{2}\left(\dot{\varepsilon}^{2}+\dot{\theta}^{2}\right)+2 \varepsilon \dot{\theta}+\frac{3}{2} \varepsilon^{2},
$$

or equivalently

$$
L=L_{C W}=\frac{1}{2}\left(\dot{\varepsilon}^{2}+\dot{\theta}^{2}\right)+(\varepsilon \dot{\theta}-\dot{\varepsilon} \theta)+\frac{3}{2} \varepsilon^{2},
$$

the two expressions differing only by the exact derivative $(\varepsilon \theta)^{\bullet}$. The corresponding equations of motion are the well known linear Clohessy-Wiltshire equations:

$$
\begin{aligned}
& \ddot{\varepsilon}=+2 \dot{\theta}+3 \varepsilon, \\
& \ddot{\theta}=-2 \dot{\varepsilon}
\end{aligned}
$$

We note that if we trace the origin of the term $3 \varepsilon^{2} / 2$, we find that two-thirds of it is due to gravity and one third to centripetal acceleration. This is because of the expression

$$
\frac{r^{2}}{2}+\frac{1}{r}=\frac{1}{2}\left(1+2 \varepsilon+\varepsilon^{2}\right)+\left(1-\varepsilon+\varepsilon^{2}\right)=\frac{3}{2}\left(1+\varepsilon^{2}\right) .
$$


The term $r^{2} / 2$ gives the centripetal acceleration, whereas the term $1 / r$ gives the inverse-square-law gravity term.

\section{The Perturbed Clohessy-Wiltshire Lagrangian}

It is apparent, from what has been seen in the previous sections that the perturbed Clohessy-Wiltshire Lagrangian

$$
L=\frac{1}{2}\left(\dot{\varepsilon}^{2}+\dot{\theta}^{2}\right)+(\varepsilon \dot{\theta}-\dot{\varepsilon} \theta)+\frac{3}{2} \varepsilon^{2}+\mu U(\varepsilon, \theta)
$$

could form a very interesting dynamical system for the study of motions near the unit-circle in a rotating frame, at least if the proper function $U(\varepsilon, \theta)$ is selected, ( $\mu$ being a small parameter, as usual). The equations of motion corresponding to (5.1) are

$$
\left\{\begin{array}{l}
\ddot{\varepsilon}=2 \dot{\theta}+3 \varepsilon+" U_{\varepsilon}, \\
\ddot{\theta}=-2 \dot{\varepsilon}+\mu U_{\theta} .
\end{array}\right.
$$

If we make no approximations at all in the potential function $U$ for the restricted three-body problem we have the following expression with three terms:

$$
U(\varepsilon, \theta)=\frac{1}{d}-(1+\varepsilon) \cos \theta-\frac{1}{1+\varepsilon} .
$$

The two last terms, expanded to the second-order in $\varepsilon$ give us:

$$
-\left(r \cos \theta+\frac{1}{r}\right)=(1+\varepsilon)(1-\cos \theta)-\varepsilon^{2}+2 .
$$

An application of this type of formulation is the Lagrangian

$$
L=L_{C W}+\mu\left[(1+\varepsilon)(1-\cos \theta)+\frac{1}{d}\right]
$$

which contains only one (first-degree) $\varepsilon$-term in the perturbation. The corresponding equations of motion are

$$
\begin{aligned}
& \ddot{\varepsilon}=+2 \dot{\theta}+3 \varepsilon+\mu(1-\cos \theta), \\
& \ddot{\theta}=-2 \dot{\varepsilon}+\mu \sin \theta \cdot\left[(1+\varepsilon)-\frac{1}{d^{3}}\right] .
\end{aligned}
$$

We assumed here that $d^{2}=2(1-\cos \theta)$.

A slightly more complicated Lagrangian would be

$$
L=L_{C W}+\mu\left[(1+\varepsilon)(1-\cos \theta)-\varepsilon^{2}+\frac{1}{d}\right],
$$


where

$$
\begin{aligned}
d^{2} & =1+(1+\varepsilon)^{2}-2(1+\varepsilon) \cos \theta=2(1+\varepsilon)(1-\cos \theta)+\varepsilon^{2}, \\
& =4(1+\varepsilon) \sin ^{2} \frac{\theta}{2}+\varepsilon^{2},
\end{aligned}
$$

or

$d^{2}=2(1+\varepsilon)(1-\cos \theta)=4(1+\varepsilon) \sin ^{2} \frac{\theta}{2}$.

or

$d^{2}=2(1-\cos \theta) 4 \sin ^{2} \frac{\theta}{2}$

according to the degree of precision that is required in $\varepsilon$. In the last formula, $d$ can vary from 0 to 2 .

If the second approximation for $d^{2}$ is used, the above Lagrangian (5.7) can also be written as

$$
L=L_{C W}+\mu\left[\frac{d^{2}}{2}+\frac{1}{d}\right] .
$$

In general the equations of motion for the last Lagrangian can be written as

$$
\begin{aligned}
& \ddot{\varepsilon}=+2 \dot{\theta}+3 \varepsilon+\mu\left(d-\frac{1}{d^{2}}\right) \frac{\partial d}{\partial \varepsilon}, \\
& \ddot{\theta}=-2 \dot{\varepsilon}+\mu\left(d-\frac{1}{d^{2}}\right) \frac{\partial d}{\partial \varepsilon} .
\end{aligned}
$$

For instance, if $d^{2}=2(1+\varepsilon)(1-\cos \theta)$, we have

$$
\frac{\partial d}{\partial \varepsilon}=\frac{1-\cos \theta}{d} ; \frac{\partial d}{\partial \theta}=\frac{(1+\varepsilon) \sin \theta}{d} \text {. }
$$

\section{A Typical Simplified Lagrangian $L_{C W}+\mu(\varepsilon, \theta)$}

As an illustration, we will now consider the approximation of the restricted problem, where the Keplerian terms as well as $U$ have all been expanded to order two only in the three variables, $\varepsilon, \dot{\varepsilon}, \dot{\theta}$, while no assumption is made on $\mu$. We also expanded $1 / d$ to order two in $\varepsilon$. We obtain then the following Lagrangian:

$$
\begin{aligned}
& L=\frac{1}{2}\left(\dot{\varepsilon}^{2}+\dot{\theta}^{2}\right)+2 \varepsilon \dot{\theta}+\frac{3 \varepsilon^{2}}{2}+\mu\left\{-\varepsilon^{2}+(1+\varepsilon)(1-\cos \theta)\right. \\
& \left.+\frac{\sqrt{2}}{2(1-\cos \theta)^{1 / 2}}\left[1-\frac{\varepsilon}{2}+\frac{\varepsilon^{2}}{8}\left(3-\frac{2}{1-\cos \theta}\right)\right]\right\}
\end{aligned}
$$

which is clearly of the form of a perturbed Clohessy-Wiltshire Lagrangian:

$$
L=\frac{1}{2}\left(\dot{\varepsilon}^{2}+\dot{\theta}^{2}\right)+2 \varepsilon \dot{\theta}+\frac{3 \varepsilon^{2}}{2}+\mu U(\varepsilon, \theta)
$$


The equations of motion for the new Lagrangian system are:

$$
\begin{aligned}
& \ddot{\varepsilon}=2 \dot{\theta}+3 \varepsilon+\mu\left\{-2 \varepsilon+(1-\cos \theta)-\frac{1}{\sqrt{2(1-\cos \theta)}}\left[\frac{1}{2}-\frac{\varepsilon}{4}\left(3-\frac{2}{1-\cos \theta}\right)\right]\right\} \\
& \ddot{\theta}=-2 \dot{\varepsilon}+\mu\left\{(1+\varepsilon) \sin \theta-\frac{\sqrt{2} \sin \theta}{4(1-\cos \theta)^{\frac{3}{2}}}\left[1-\frac{\varepsilon}{2}+\frac{3 \varepsilon^{2}}{8}\left(1-\frac{2}{1-\cos \theta}\right)\right]\right\} .
\end{aligned}
$$

It is not difficult to show, by letting $\dot{\varepsilon}=\ddot{\varepsilon}=\dot{\theta}=\ddot{\theta}=0$, that the Lagrange points $L_{3}, L_{4}$, and $L_{5}$ still exist with the coordinates:

$$
\begin{array}{ll}
L_{3}: \varepsilon=\frac{-7 \mu}{12-7 \mu}, \theta=180^{\circ}, \\
L_{4}: \varepsilon=0 & , \theta=+60^{\circ}, \\
L_{5}: \varepsilon=0 & , \theta=-60^{\circ} .
\end{array}
$$

\section{The Second Approximate Model}

For many situations $\mu<<1$, so the additional assumption of neglecting terms of order 3 and higher in $\mu, \varepsilon$ and $\dot{\theta}$ will be made. This simplifies the Lagrangian to

$$
L=\frac{1}{2}\left(\dot{\varepsilon}^{2}+\dot{\theta}^{2}\right)+2 \varepsilon \dot{\theta}+\frac{3 \varepsilon^{2}}{2}+\mu(1+\varepsilon)(1-\cos \theta)+\frac{\mu}{[2(1-\cos \theta)]^{\frac{1}{2}}}\left(1-\frac{\varepsilon}{2}\right)(28)
$$

and the equations of motion:

$$
\begin{aligned}
& \ddot{\varepsilon}=2 \dot{\theta}+3 \varepsilon+\mu(1-\cos \theta)\left[1-\frac{\sqrt{2}}{4(1-\cos \theta)^{\frac{3}{2}}}\right], \\
& \ddot{\theta}=-2 \dot{\varepsilon}+\mu\left[(1+\varepsilon) \sin \theta-\frac{\sqrt{2} \sin \theta}{4(1-\cos \theta)^{\frac{3}{2}}}\left(1-\frac{\varepsilon}{2}\right)\right] .
\end{aligned}
$$

The Lagrange points are as before.

\section{The Unit-Circle Symmetry in Polar Coordinates}

We can easily show that the perturbed Clohessy-Wiltshire equations

$$
\begin{aligned}
& \ddot{\varepsilon}=+2 \dot{\theta}+3 \varepsilon+\mu U_{\varepsilon}(\varepsilon, \theta) \\
& \ddot{\theta}=-2 \dot{\varepsilon}+\mu U_{\theta}(\varepsilon, \theta)
\end{aligned}
$$

exhibit a remarkable symmetry condition, under some special constraints on the potential function $U(\varepsilon, \theta)$. In particular, if we assume that $U_{\varepsilon}(\varepsilon, \theta)$ is an odd function of $\varepsilon$ and $U_{\theta}(\varepsilon, \theta)$ an even function of $\varepsilon$, then, the substitutions

$$
\varepsilon \rightarrow-\varepsilon ; \theta \rightarrow \theta ; \dot{\varepsilon} \rightarrow \dot{\varepsilon} ; \dot{\theta} \rightarrow \dot{\theta} ; \ddot{\varepsilon} \rightarrow \ddot{\varepsilon} ; \ddot{\theta} \rightarrow \ddot{\theta}
$$

leave the above equations of motion invariant. Indeed, we see that this substitution changes all the signs of the terms of the first equation, while it produces 
no change at all to the second equation of motion. This results in a mirror-image property: to every solution corresponds a mirror image solution relative to the horizontal axis in the $(\theta, \varepsilon)$-plane, (the axis $\varepsilon=0$ ). In other words, to every arc of solution outside the unit-circle corresponds another arc of solution inside the unit circle. This property is thus very similar to the mirror-image theorem which is well known in the restricted problem, relative to the syzygy-axis. This symmetry property can also be used to find a special kind of periodic solutions: A sufficient condition of periodicity is that the solution must have two orthogonal intersections with the unit-circle. In other words the solution should have two points with initial and final conditions of the form

$$
\left(0, \theta_{0}, \dot{\varepsilon}_{0}, 0\right)
$$

these two points being separated by a half-period. Another fact that can be noted in relation to these properties is that we could use the symmetry line $\varepsilon=0$, (the unit-circle) as the line of sections for the construction of Poincare maps in the $(\theta, \dot{\theta})$-plane.

As for the potential function $U(\varepsilon, \theta)$, we see that it clearly must satisfy the general condition of being an even function of the variable $\varepsilon$. Let us mention that this symmetry property does not hold for the true untruncated restricted problem. We also note that the condition for the unit-circle symmetry does hold for the simplified systems of the form

$$
\begin{aligned}
& \ddot{\varepsilon}=+2 \dot{\theta}+3 \varepsilon, \\
& \ddot{\theta}=-2 \dot{\varepsilon}+B\left(\theta, \varepsilon^{2}\right)
\end{aligned}
$$

where $B$ is an arbitrary function not depending on $\varepsilon$ or even in $\dot{\varepsilon}$.

The above considerations are completely independent of the $\theta$-dependence of the potential $U(\varepsilon, \theta)$. However, the standard mirror-image theorem with the syzygy axis always holds: orbits with initial values $\theta_{0}=0$ or $\pi$ and $\dot{\varepsilon}_{0}=0$ are symmetric with respect to the syzygy-line (which joins the two primaries).

As for the distance $d$ between the second primary and the satellite, the two following approximations

$$
d^{2}=\varepsilon^{2}+2(1-\cos \theta) ; d^{2}=2(1-\cos \theta)
$$

both satisfy the unit-circle symmetry criterion.

As an example of a potential function which would be a reasonable approximation for the restricted three-body problem and which at the same time satisfies the unit-circle symmetry condition, we propose

$$
U(\varepsilon, \theta)=-(1+\cos \theta)-\varepsilon^{2}+\frac{1}{\sqrt{\varepsilon^{2}+2(1-\cos \theta)}},
$$


which has the derivatives

$$
U_{\varepsilon}=-\varepsilon\left(2+\frac{1}{d^{3}}\right) ; U_{\theta}=+\sin \theta\left(1-\frac{1}{d^{3}}\right),
$$

where $d$ represents the first of the two distance approximations (8.5) given above.

\section{The Case where $U$ depends on $\theta$ only}

An interesting special case corresponds to the situation where the function $U$, present in the perturbed Clohessy-Wiltshire Lagrangian contains explicitly only the variable $\theta$. We have then the equations of motion

$$
\ddot{\varepsilon}=+2 \dot{\theta}+3 \varepsilon ; \ddot{\theta}=-2 \dot{\varepsilon}+\mu U_{\theta},
$$

so that the perturbation acts only on the theta-equation.

As an illustration, we mention the Lagrangian

$$
L=L_{C W}+\mu\left[(1-\cos \theta)+\frac{1}{\sqrt{2(1-\cos \theta)}}\right]=L_{C W}+\mu\left[2 \sin ^{2} \frac{\theta}{2}+\frac{1}{2 \sin \frac{\theta}{2}}\right]
$$

The equations of motion derived from this Lagrangian are

$$
\ddot{\varepsilon}=+2 \dot{\theta}+3 \varepsilon ; \ddot{\theta}=-2 \dot{\varepsilon}+\mu\left(1-\frac{1}{8 \sin ^{3}(\theta / 2)}\right) \sin \theta
$$

In the case where we would have only motions which are extremely close to the unit circle, we could assume that $\ddot{\varepsilon}=0$, so that, by differentiation of the right-hand side of the first equation $\dot{\varepsilon}=-2 \ddot{\theta} / 3$, (Yoder et al., 1983). The second equation becomes thus

$$
\ddot{\theta}=\frac{+4 \ddot{\theta}}{3}+\mu U_{\theta}
$$

which is equivalent to

$$
\ddot{\theta}=-3 \mu U_{\theta} \text {. }
$$

Note that this equation has the Lagrangian

$$
L=\frac{1}{2} \dot{\theta}^{2}-3 \mu U(\theta) \text {. }
$$

We believe that this Lagrangian is somewhat more realistic than the Lagrangian which is obtained by setting $\varepsilon=\dot{\varepsilon}=0$ in (5.1):

$$
L=\frac{1}{2} \dot{\theta}^{2}+\mu U(\theta) \text {. }
$$


As an illustration we mention again the case where

$$
\begin{aligned}
U(\theta) & =(1-\cos \theta)+(2(1-\cos \theta))^{-1 / 2}=(1-\cos \theta)+\frac{1}{d} \\
& =2 \sin ^{2} \frac{\theta}{2}+\left(2 \sin \frac{\theta}{2}\right)^{-1}
\end{aligned}
$$

which will clearly result in a perturbed pendulum problem.

The Lagrangian

$$
L=\frac{1}{2} \dot{\theta}^{2}-3 \mu U(\theta)
$$

gives us the equation of motion

$$
\ddot{\theta}=-3 \mu\left(1-\frac{1}{8 \sin ^{3}(\theta / 2)}\right) \sin \theta
$$

or

$$
\ddot{\theta}=-3 \mu\left[1-\frac{2}{\sqrt{8(1-\cos \theta)^{3}}}\right] \sin \theta
$$

which has two important terms: the first term $(-3 \mu \sin \theta)$ gives the usual type of pendulum motion. However, the second term has the opposite sign. In these formulations, the radial variable $\varepsilon$ can be recovered by using Yoderis equation $\varepsilon=2 \dot{\theta} / 3$.

We note that Yoder et al. (1983) has used an equation of the general form of a perturbed pendulum

$$
\ddot{\theta}=-3 \mu \sin \theta+\cdots
$$

in his work on the co-orbital satellites of Saturn.

The above dynamical system being conservative, it has the Energy equation:

$$
E=\frac{1}{2} \dot{\theta}^{2}+3 \mu\left[2 \sin ^{2} \frac{\theta}{2}+\frac{1}{2 \sin (\theta / 2)}\right]=\text { Constant. }
$$

It will be convenient to define the Jacobi constant $C$ for this problem, with the equation

$$
E=\frac{3}{2} \mu C
$$

It is clear that the present dynamical system is integrable. In fact the above energy equation gives the solution ( $\dot{\theta}$ as a function of $\theta$, for instance). 
which has the derivatives

$$
U_{\varepsilon}=-\varepsilon\left(2+\frac{1}{d^{3}}\right) ; U_{\theta}=+\sin \theta\left(1-\frac{1}{d^{3}}\right) \text {, }
$$

where $d$ represents the first of the two distance approximations (8.5) given above.

\section{The Case where $U$ depends on $\theta$ only}

An interesting special case corresponds to the situation where the function $U$, present in the perturbed Clohessy-Wiltshire Lagrangian contains explicitly only the variable $\theta$. We have then the equations of motion

$$
\ddot{\varepsilon}=+2 \dot{\theta}+3 \varepsilon ; \ddot{\theta}=-2 \dot{\varepsilon}+\mu U_{\theta},
$$

so that the perturbation acts only on the theta-equation.

As an illustration, we mention the Lagrangian

$$
L=L_{C W}+\mu\left[(1-\cos \theta)+\frac{1}{\sqrt{2(1-\cos \theta)}}\right]=L_{C W}+\mu\left[2 \sin ^{2} \frac{\theta}{2}+\frac{1}{2 \sin \frac{\theta}{2}}\right]
$$

The equations of motion derived from this Lagrangian are

$$
\ddot{\varepsilon}=+2 \dot{\theta}+3 \varepsilon ; \ddot{\theta}=-2 \dot{\varepsilon}+\mu\left(1-\frac{1}{8 \sin ^{3}(\theta / 2)}\right) \sin \theta
$$

In the case where we would have only motions which are extremely close to the unit circle, we could assume that $\ddot{\varepsilon}=0$, so that, by differentiation of the right-hand side of the first equation $\dot{\varepsilon}=-2 \ddot{\theta} / 3$, (Yoder et al., 1983). The second equation becomes thus

$$
\ddot{\theta}=\frac{+4 \ddot{\theta}}{3}+\mu U_{\theta}
$$

which is equivalent to

$$
\ddot{\theta}=-3 \mu U_{\theta} \text {. }
$$

Note that this equation has the Lagrangian

$$
L=\frac{1}{2} \dot{\theta}^{2}-3 \mu U(\theta) \text {. }
$$

We believe that this Lagrangian is somewhat more realistic than the Lagrangian which is obtained by setting $\varepsilon=\dot{\varepsilon}=0$ in (5.1):

$$
L=\frac{1}{2} \dot{\theta}^{2}+\mu U(\theta) .
$$


As an illustration we mention again the case where

$$
\begin{aligned}
U(\theta) & =(1-\cos \theta)+(2(1-\cos \theta))^{-1 / 2}=(1-\cos \theta)+\frac{1}{d} \\
& =2 \sin ^{2} \frac{\theta}{2}+\left(2 \sin \frac{\theta}{2}\right)^{-1}
\end{aligned}
$$

which will clearly result in a perturbed pendulum problem.

The Lagrangian

$$
L=\frac{1}{2} \dot{\theta}^{2}-3 \mu U(\theta)
$$

gives us the equation of motion

$$
\ddot{\theta}=-3 \mu\left(1-\frac{1}{8 \sin ^{3}(\theta / 2)}\right) \sin \theta
$$

or

$$
\ddot{\theta}=-3 \mu\left[1-\frac{2}{\sqrt{8(1-\cos \theta)^{3}}}\right] \sin \theta
$$

which has two important terms: the first term $(-3 \mu \sin \theta)$ gives the usual type of pendulum motion. However, the second term has the opposite sign. In these formulations, the radial variable $\varepsilon$ can be recovered by using Yoderis equation $\varepsilon=2 \dot{\theta} / 3$.

We note that Yoder et al. (1983) has used an equation of the general form of a perturbed pendulum

$$
\ddot{\theta}=-3 \mu \sin \theta+\cdots
$$

in his work on the co-orbital satellites of Saturn.

The above dynamical system being conservative, it has the Energy equation:

$$
E=\frac{1}{2} \dot{\theta}^{2}+3 \mu\left[2 \sin ^{2} \frac{\theta}{2}+\frac{1}{2 \sin (\theta / 2)}\right]=\text { Constant. }
$$

It will be convenient to define the Jacobi constant $C$ for this problem, with the equation

$$
E=\frac{3}{2} \mu C \text {. }
$$

It is clear that the present dynamical system is integrable. In fact the above energy equation gives the solution ( $\dot{\theta}$ as a function of $\theta$, for instance). 
The most important property of the present system is the existence of three equilibrium solutions. Their existence can be justified analytically, by finding the roots of the equation

$$
\sin \theta \cdot\left[1-1 /\left(8 \sin ^{3} \frac{\theta}{2}\right)\right]=0 .
$$

The first root corresponds to $\theta=0$. However this corresponds to the singularity at the center of the smaller primary at $(1,0)$. By writing the equation of motion as

$$
\ddot{\theta}=-6 \mu \cos \frac{\theta}{2}\left[\sin \frac{\theta}{2}-1 /\left(8 \sin ^{2} \frac{\theta}{2}\right)\right]
$$

we see that, at $\theta=0, \ddot{\theta}=6 \mu\left(0-\frac{1}{0}\right) \simeq+\infty$. This point $\theta=0$ is thus a repulsive point. The other root is $\theta=\pi$, which is an unstable equilibrium point: $L_{3}$, one of the three collinear Lagrange points.

Finally we have the two roots $\sin \frac{\theta}{2}=\frac{1}{2}$, which are thus $\theta= \pm 60^{\circ}$, the two triangular Lagrangian points $L_{4}$ and $L_{5}$. It is remarkable that in this dynamical system, which is an extreme simplification of the restricted problem, three of the five Lagrange points still survive !

\section{References}

Deprit, A. and Henrard, J.: 1978, "A Manifold of Periodic Orbits", Advances in Astronomy and Astrophysics, Vol. 6, Academic Press, New York.

Deprit, A.: 1966, "Motion in the Vicinity of the Triangular Libration Centers", Lectures in Applied Mathematics, Vol. 6, American Mathematical Society.

Deprit, A., Henrard, J., Palmore, J. and Price, J F.: 1969, "The Trojan Manifold in the System Earth-Moon", Mon. Not. R. Astr. Soc., 137, 311-335.

Dermott, S.F. and Murray, C.D.: 1981, "The Dynamics of Tadpole and Horseshoe Orbits: I. Theory", Icarus, 48, 1-11.

Dermott, S. F. and Murray, C.D.: 1981, "The Dynamics of Tadpole and Horseshoe Orbits: II. The Coorbital Satellites of Saturn", Icarus, 48, 12-22.

Garfinkel, B.: 1977, "Theory of the Trojan Asteroids, Part I", Astron. J., 82, 368-379.

Garfinkel, B.: 1975, "An Extended Ideal Resonance Problem", Celestial Mechanics, 12, 203-214.

Garfinkel, B.: 1978, "Theory of the Trojan Asteroids. Part II", Celest. Mech., 18, 259-275.

Giacaglia, G. E. O.: 1976, comments on the paper by Boris Garfinkel: "An Extended Ideal Resonance Problem", Celestial Mechanics, 13, 515-516.

Harrington, R.S. and Seidelmann P.K.: 1981, "The Dynamics of the Saturnian Satellites 1980S1 and 1980S3", Icarus, 47 , 97-99

Konopliv, A. S.: 1986, “Theory of Coorbital Motion", Ph.D. dissertation, Univ. of Texas at Austin.

Salo, H. and Yoder C F.: 1988, "The Dynamics of Coorbital Satellite Systems", A.\& A., 205, 309-327

Yoder, C. F., G. Colombo, S. P. Synnott, and K. A. Yoder, 1983, “Theory of Motion of Saturn's Coorbiting Satellites", Icarus, 53, 431-443. 